\title{
EDITORIAL
}

\section{DESAFÍOS PARA EL DESARROLLO Y FORTALECIMIENTO DE LA INVESTIGACIÓN EN ENFERMERÍA}

\author{
CHALLENGES FOR NURSING RESEARCH DEVELOPMENT AND \\ STRENGTHENING \\ MSc María Consuelo Cerón Mackay \\ Decana. Facultad de Enfermería y Obstetricia, Universidad de los Andes
}

Es indudable que la búsqueda de nuevo conocimiento es fundamental para el desarrollo de cualquier profesión. Asimismo, una profesión que no valida y renueva su cuerpo de conocimientos, y en su defecto se nutre de otras disciplinas, está destinada a desaparecer. En enfermería ha existido un avance importante en las últimas décadas en la producción científica, principalmente en lo referente al desarrollo disciplinar; sin embargo, existen temas pendientes como la consolidación, difusión y aplicación de la investigación en el cuidado directo $(1,2)$.

Dentro de los principales desafíos que enfrentamos en el desarrollo de la investigación en enfermería es de qué manera esta expansión del conocimiento pueda ser llevada y aplicada en la práctica clínica y ser de utilidad para la toma de decisiones respecto del cuidado directo o indirecto de los pacientes (2). No tiene ninguna utilidad que los resultados se mantengan entre las cuatro paredes de la universidad o en revistas científicas de difícil acceso. Esta afirmación conlleva a dos preguntas críticas, la primera es sobre qué investigamos y la segunda es cómo logramos que efectivamente las enfermeras clínicas tengan acceso a ella y la utilicen en su práctica diaria.

Con respecto a la primera pregunta sobre qué estudiar, parece de toda lógica responder lo que preocupa a la enfermera clínica. Sin embargo, los desafíos actuales en salud obligan a ampliar esta mirada e intentar dar solución a los problemas macro del sistema de salud en general, que sin duda preocupan a la enfermera. A modo de ejemplo, y teniendo en cuenta los lineamientos estratégicos en salud global y local, las investigaciones que aporten a mejorar los resultados de salud de los pacientes, a mejorar la experiencia en salud y a reducir los costos en la atención de salud son prioritarias. Por su parte, el National Institute of Nursing Research, en su plan estratégico 2016 hace un llamado a considerar la importancia de la tecnología dentro de las líneas de investigación científica, como por ejemplo las mediciones 
de parámetros fisiológicos de pacientes crónicos o posalta a través de la teleenfermería o aplicaciones móviles. Asimismo, demanda el aprovechar al máximo la información contenida en las fichas electrónicas (3). Sobre este punto es necesario detenerse un momento debido a que el futuro de la investigación en salud está en los Big Data que se generan a partir de los registros electrónicos de pacientes (3). El Big Data consiste en un gran volumen de datos complejos de pacientes que requieren de análisis estadísticos avanzados y de los cuales emerge el conocimiento para transferirlo a la práctica clínica, en vez de testear la realidad como lo hemos venido haciendo hasta ahora (3).

A nivel nacional existen bases de datos complejas que contienen gran cantidad de información de pacientes y que pueden ser interesantes para la investigación en enfermería. Por ejemplo, los Grupos Relacionados por el Diagnóstico (GRD), implementados en todos los hospitales de alta complejidad y en el sector privado, relacionan la atención de salud recibida por el paciente con el pago por dicha atención. Los GRD contienen información sociodemográfica de los pacientes, así como la vía de ingreso, comorbilidades, complicaciones, tiempo de estadía hospitalaria y vía de egreso, entre otros. Asimismo, y como parte de la estrategia sobre Sistemas de Información de la Red Asistencial (SIDRA) se han implementado en más de 600 centros de salud en Atención Primaria sistemas de registro electrónico de pacientes con distintos grados de avance. Estos registros del historial de las atenciones de los usuarios contienen información relevante como morbilidades, dispensación de fármacos, episodios agudos y factores de riesgo, por mencionar algunos. Otra fuente importante de almacenamiento de datos y estrechamente relacionada al cuidado de enfermería la constituye el Sistema de Vigilancia y reporte de Eventos Adversos (EA), que permite no solo hacer un seguimiento de los EA sino que también un análisis complejo sobre la ocurrencia de los EA en beneficio de los pacientes.

Las investigaciones en enfermería que utilicen la gran cantidad de datos acumulados es una oportunidad de ampliar considerablemente el conocimiento enfermero, en estrecha sintonía con las necesidades de los pacientes y los problemas macro del sistema de salud y en consecuencia aportar con investigación de alto impacto social.

Finalmente, respecto de cómo podemos efectivamente hacer llegar la investigación a las enfermeras asistenciales desde la academia, debemos considerar que la investigación traslacional es uno de los principales focos de los fondos concursables, ya que en términos de costo-efectividad permiten generar resultados para una atención de salud basada en evidencia. Por tanto, si queremos aumentar la proporción de fondos concursables adjudicados por enfermería, tenemos que pensar en investigaciones con aplicación clínica y cuyo propósito sea mejorar los resultados de salud de los pacientes (4). Desde la perspectiva de las enfermeras asistenciales, es necesario superar algunas barreras para transformarse en consu- 
midoras de investigación. Una de estas barreras mencionadas por ellas mismas es la falta de tiempo, el difícil acceso que tiene a revistas indexadas, como asimismo la capacidad de lectura crítica de sus resultados. Refieren no contar con las competencias metodológicas y estadísticas para entender, analizar críticamente y menos aplicar los resultados de las investigaciones. Esto se traduce en que no son buenas consumidoras de investigación y mucho menos agentes replicadores de la evidencia generada, por ejemplo, a través de la elaboración de protocolos, guías clínicas o planes de cuidado. Esto sin contar con que el 80,2\% de las publicaciones indexadas en la Web of Science son en inglés (5), y en los currículos de enfermería la introducción del inglés es reciente.

Mejorar la difusión y acercar el conocimiento de la evidencia a la práctica asistencial es un desafío que requiere de un abordaje conjunto entre el mundo asistencial y académico. Sin considerar que tenemos pendiente, en este abordaje conjunto, la difusión social (2) a través de la prensa, de la investigación en enfermería, ya que el rol social de nuestra profesión nos obliga a comunicar a la sociedad lo que sabemos hacer: cuidar de la salud de las personas.

\section{REFERENCIAS}

1. Varela MD, Sanjurjo ML, Blanco F. La investigación en enfermería. Rol de la enfermería. Asociación Española de Enfermería en Urología. 2012; 121: 19-21.

2. Vélez E. Investigación en Enfermería, fundamento de la disciplina. Rev Adm Sanit. 2009; 7(2): 341-56.

3. Westra BL, Sylvia M, Weinfurter EF, Pruinelli L, Park JI, Dodd D et al. Big Data science: a literature review of nursing research exemplars. Nurs Outlook [Internet]. 2016 [citado 15 dic 2016]: [13 p.]. Disponible en: http://www.nursingoutlook.org/

4. Christian B. Translational Research - The Panoramic Scope of Pediatric Nursing and New Horizons for Improving the Health of Children, Adolescents, and their Families. J Pediatr Nurs. En prensa 2016.

5. Cáceres MC, Guerrero-Martín J, González-Morales B, Pérez-Civantos DV, Carreto-Lemus MA, Durán-Gómez N. Impact factor evolution of nursing research journals: 2009-2014. Nurs Outlook. En prensa 2017. 